International Journal on Cybernetics \& Informatics (IJCI) Vol. 5, No. 2, April 2016

\title{
BORDER SECURITY ROBOT
}

\author{
Minni Mohan ${ }^{1}$ And Siddharth Shelly ${ }^{2}$ \\ ${ }^{1}$ Department of electronics and communication, M.A College of Engineering, \\ Kothamangalam,A P J Abdul Kalam Technological University, Kerala, India \\ ${ }^{2}$ Associate Prof, Department of electronics and communication Engineering, \\ Mar Athanasius College of Engineering, Kothamangalam, Kerala, India
}

\begin{abstract}
The ordinary border patrol system suffers from intensive human involvement. Recently unmanned border patrol system consist of high tech devices, like unmanned aerial vehicles, unattended ground sensors, and surveillance towers equipped with wireless camera. However, any single technique encounters inextricable problems, such as high false alarm rate and line of sight constrains. There require a coherent system that co-ordinates various technologies to improve the system accuracy. In this project general idea of boarder security robot, wireless sensor network architecture for border patrol system, is introduced. Border security robot utilize a PIR sensor for human detection, a metal detector to detect the presence of explosives and a wireless camera for monitoring the scenario continuously at the remote station. Mechanical control of robotic vehicle along with robotic arm can be done from the remote station. This is initiated with a Bluetooth module.
\end{abstract}

\section{KEYWORDS}

PIC, PIR, Metal detector, Wireless camera

\section{INTRODUCTION}

Border patrol systems have recently achieved interest to address the concerns about national security. The major problem in protecting long stretches of borders is the need for large human involvement in patrolling the premises. In our border patrol system consists of security checkpoints and border troops. All vehicle traffic is need to stop in security check points which are set up on the international roads to detect and apprehend illegal aliens, drugs, and other illegal activity. The border troop watches and maintain control in a specific section of the border. The troops patrol the border according to predetermined route and time interval ${ }^{[1]}$. Under the conventional border patrol system, even modest-sized areas require large human resources if manual patrolling is considered alone. To monitor the border in real-time with accuracy and minimize the need for human support, multiple surveillance technologies, which complement each other are required. To address the challenges still facing by the existing surveillance techniques, we introduce Border security robot, a new border patrol system framework based on hybrid wireless sensor networks, which can accurately detect the border intrusion with minimum human involvements. Border security robot utilizes the PIR sensor for human detection and a metal detector for explosive detection. Also a wireless camera is used to continuously monitor the border. While the potential benefits of Border security are significant, several research challenges need to be addressed before a practical realization. In this project, a framework to deploy and operate Border Sense for border patrol is described.

DOI: 10.5121/ijci.2016.5230 


\section{Technology Used}

\subsection{Bluetooth Technology}

The convergence of computing and communications has led to the development of Bluetooth technology. Taking the short-range wireless data usage to a new level, this technology is predicted to dominate both the home and business market. Bluetooth is a wireless technology standard for exchanging data over short distances (using short-wavelength radio transmissions in the ISM band 2400-2480 MHz) from fixed and mobile devices, creating personal area networks with high levels of security. It was originally conceived ${ }^{[2]}$ as a wireless alternative to RS-232 data cables which is Created by telecom vendor Ericsson in 1994. It can connect several devices; problems of synchronization can be overcome.

In this project Bluetooth technology a wireless protocol that used to connects robotic side and personal computer in remote station. Configuring Bluetooth module all communications between remote station and robotic side can be done.

\subsection{Infrared Technology}

In order to understand what thermal imaging is, it is important to understand something about light. The total amount of energy in a light wave is related to its wavelength and shorter wavelengths have higher energy. Of visible light, the most energy for violet, and the least energy for red. Just next to the visible light spectrum is called as infrared spectrum. Shown in fig.1

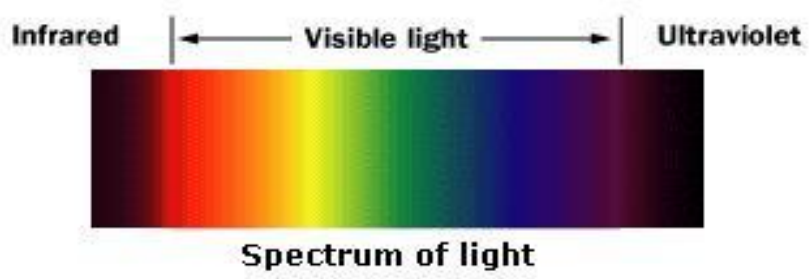

Fig.1.Spectrum of light

Infrared light can be split into three categories:

\section{Near-infrared (near-IR)}

It closest to visible light, near-Infrared has wavelengths that range from meters 700 billionths to 1,300 billionths of a meter.

\subsubsection{Mid-infrared (mid-IR)}

Mid-Infrared has wavelengths ranging from 1300 to 3000 billionths of a meter. Both nearInfrared and mid-Infrared are used in variety of electronic devices. 


\subsubsection{Thermal-infrared (thermal-IR)}

It Occupying the largest part of the infrared spectrum, thermal-IR has wavelengths ranging from 3000-30000 billionths of a meter (3 microns to over 30 microns).

In this border security robot continuous monitoring of border locations is done by using thermal imaging camera, which use infrared technology to capture images. The main difference between thermal-Infrared and the other two range is that thermal-Infrared is emitted by an object instead of reflected off it. In an object some process happening at the atomic level leads the emission of infrared lights. The signal-processing unit sends the information to the display, depending on the intensity of the infrared emission it appears as various colors. The combination of all the emissions coming from all of the elements used to creates the image

\section{Proposed Model Explanation}

\subsection{Robot}

The module consists of an Embedded System device which includes a central Microcontroller with a PIR sensor, metal detector and a Bluetooth interface. Two L293D driver ICs are used to drive the four motors. Drivers ensure the proper working voltage for DC motor and also protect the microcontroller from being harmed due to the back emf generated in the motor. Out of four DC motors, two are for the mechanical control of robotic vehicle and two are for robotic arm. The four mechanical controls that is being given to the robotic vehicle are forward, backward, right and left and that for robotic arm are up, down, expand and contract. The analog output of metal detector is connected to the controller through the inbuilt ADC in PIC and PIR sensor is connected to the external interrupt pin. Whenever an intruder or explosive is detected, PIC initiates the corresponding warning message transfer through Bluetooth module. Block diagram is shown in fig. 2

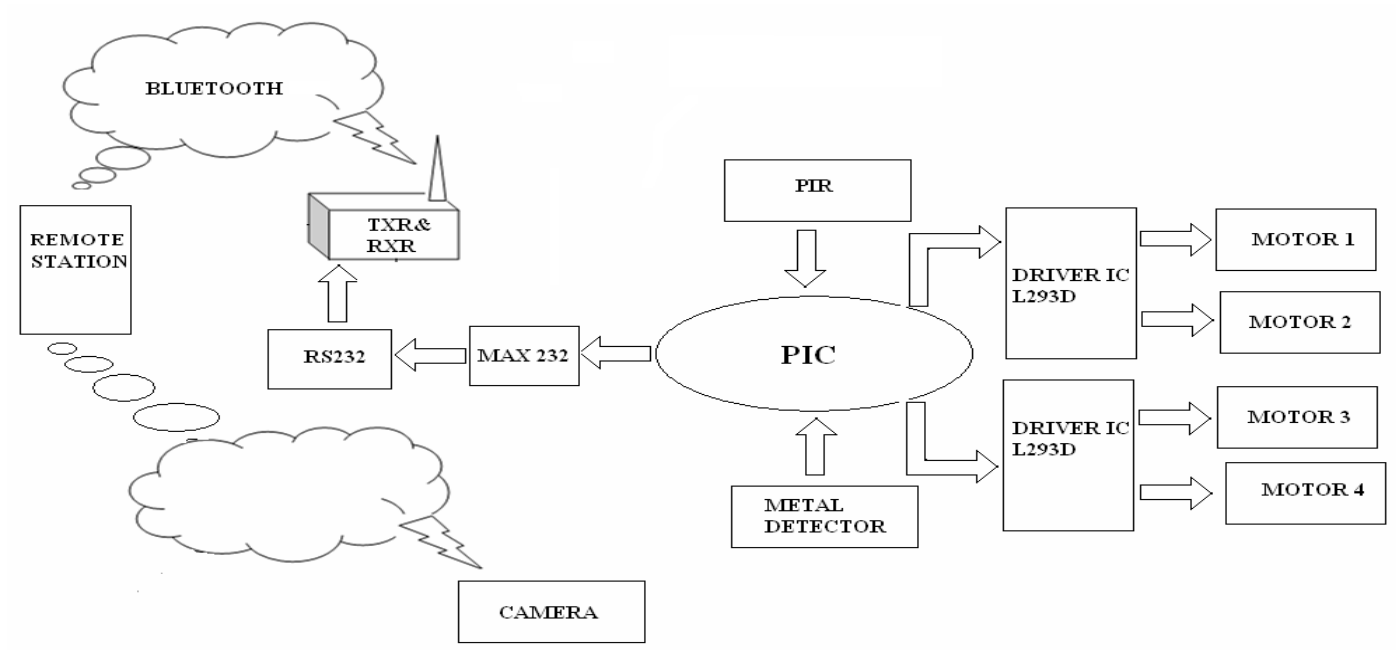

Fig.2 Block diagram 


\subsection{Pc Side}

PC is connected to the robot through the inbuilt Bluetooth module. Whenever a warning message is received, corresponding secure measures can be taken by controlling both the robotic arm and vehicle by monitoring the border through wireless camera. With the help of wireless camera border scenario can be continuously monitor in control section

\section{Flow Chart}

Software part in remote station and robotic side are start by some initializations, which is provided for configuring wireless modules. In remote station configurations are set, with the use of visual basic GUI robotic part can be controlled. Both arm control and robotic motions can be controlled from remote station by using command button in GUI interface or by using configured icons in keyboard. For that icons in keyboard S, G, C, E and H, M, K, U configured. At the same time border scenario can be monitored using wireless camera, which work with infrared technology. It provides good quality images in nights, in presence of fog, mist etc. Thermal imaging camera is capturing their images by using infrared lights coming from each and every hot body in surrounding. So it is possible to find any hidden intruders in the surrounding from thermal imaging camera captured video. Remote station gets complete visualization of border scenario with high quality than conventional manual patrolling. If any intruder or explosive is found by robot the moment itself it inform to the control section of remote station. In remote station software is continuously checking the massages coming from robots and which is displayed in GUI and informs the authorized persons by alarm. Remote station flow chart is shown in fig.3.

In robotic side after initialization controller provided in robotic side continuously checking the output of PIR sensor, metal detector and remote station information's. If the PIR sensor output is high means "an intruder found", Controller send this information to remote station through Bluetooth interface. But any explosive is detected, which is sensed by controller using an interrupt (INTF) and a message "bomb detected" is send to remote station. Information's from remote stations are also received by monitoring RCIF interrupt. According to the information robot movements and its arm can be controlled. Flow chart explanation of robotic side is shown in fig. 4

\subsection{Remote Station}

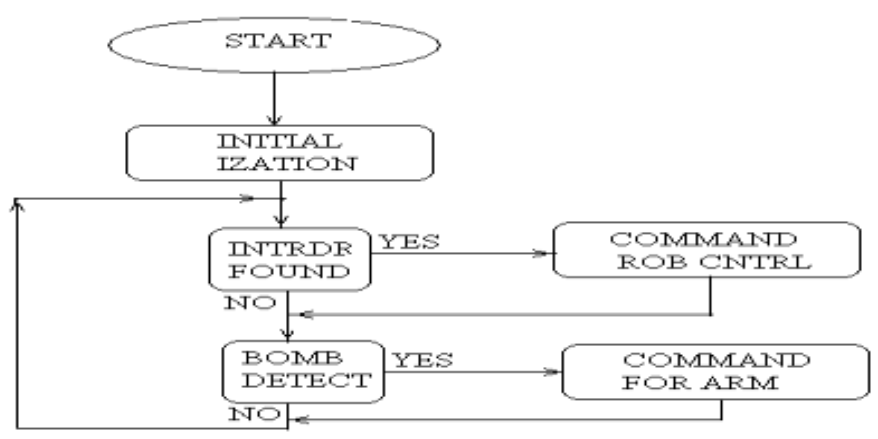

Fig3.Flow chart Remote Station 


\subsection{Robotic Side}

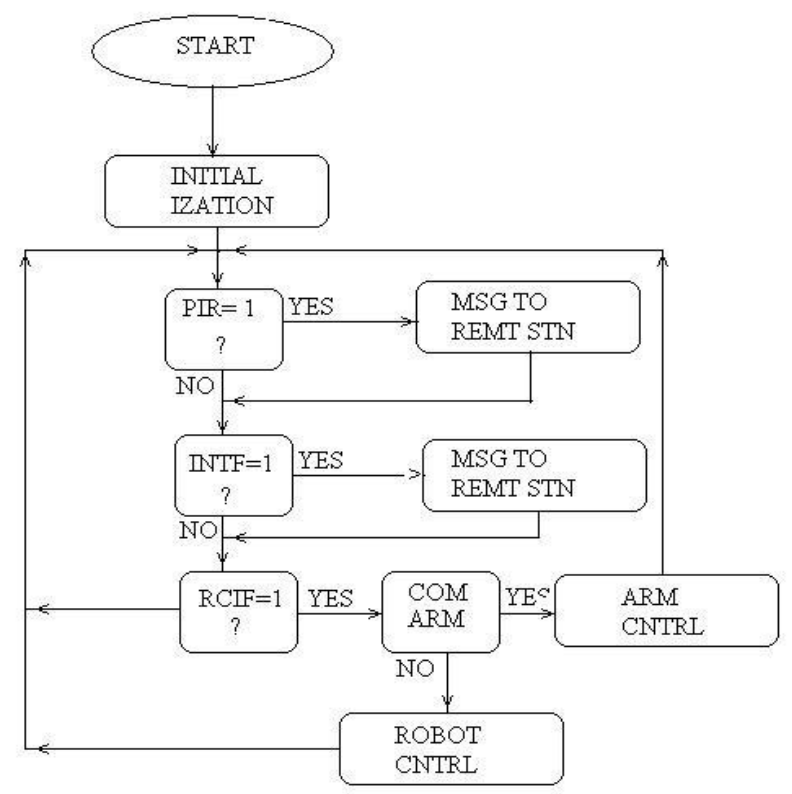

Fig.4.Flow chart -robotic side

\section{Discussions And Result}

\subsection{Mplab Software:}

It is possible to create the source files in a text editor like Notepad. Then run the Compiler on each $\mathrm{C}$ source file, which specifying a list of controls and run the Assembler on each Assembler source file, which specifying another list of controls, run either the Library Manager or Linker (again specifying a list of controls) and finally use the Object-HEX Converter to convert the Linker output file to an Intel Hex File. Once that completed the Hex File can download to the target hardware and can be debugged. Alternatively MPLAB can be used to create source files, which automatically compile, link and convert using options set with an easy to use user interface and finally simulate on the hardware with access to $\mathrm{C}$ variables and memory. Unless you have to use the tolls on the command line. MPLAB Greatly simplifies the process of creating and testing an embedded application.

\subsection{VISUAL BASIC 6.0}

Microsoft Visual basic 6.0 is the application software used to interface Bluetooth to PC. Robot.exe file on execution opens a window as shown in figure. Visual Basic is a thirdgeneration event-driven programming language and (IDE) integrated development environment ${ }^{[3]}$. It from Microsoft for its COM programming model and it is first released in 1991. Visual Basic is designed to be comparatively easy to learn and use. It was derived from BASIC 
and it enables the rapid application development of graphical user interface applications, which access to databases using Data Access Objects and Remote Data Objects.

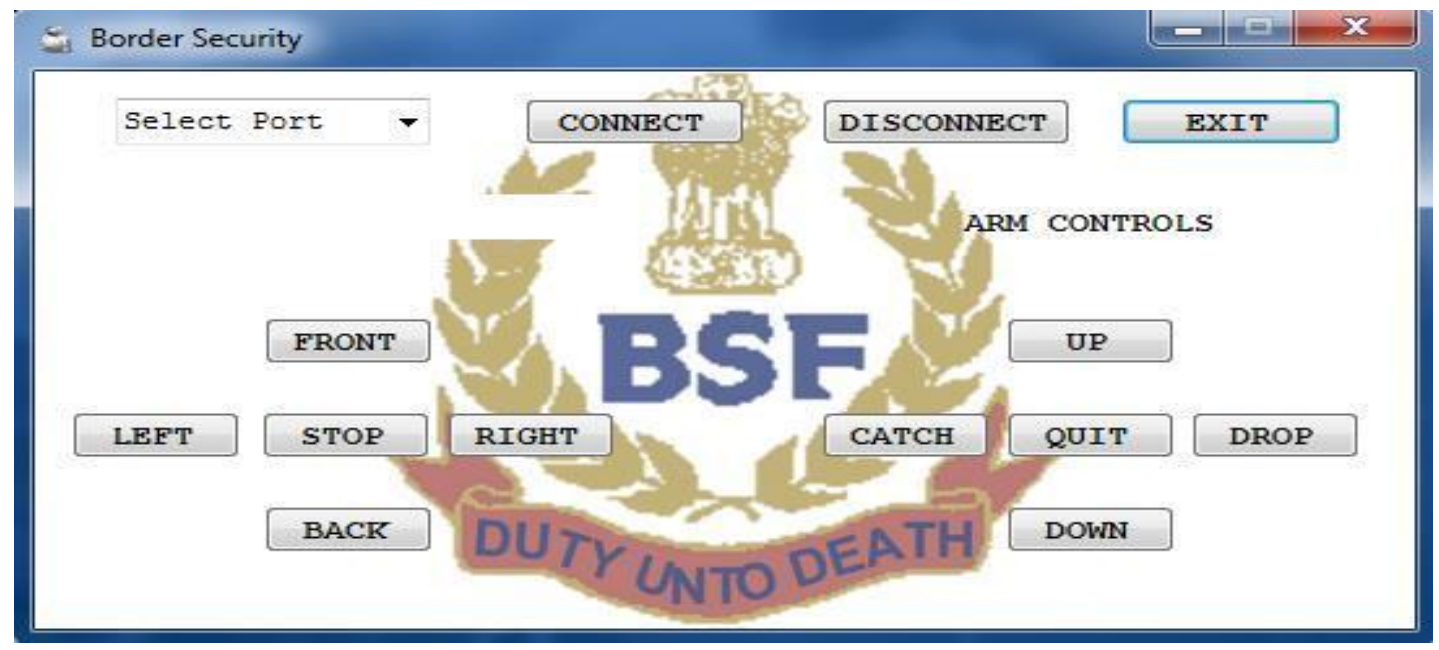

Fig.5 VB application home page

In the select port combo box all available com ports of a PC will be shown in fig 5. From that select the appropriate com port and then press the connect button. In this project comport 13 is configure to connect with Bluetooth module. This will connect the Bluetooth module in PC and Bluetooth module in robot. Whenever an intruder or explosive detected, the message "an intruder detected" and "bomb detected" will be displayed in the dialogue box of the above shown form. FRONT, BACK, LEFT, RIGHT and STOP are the command buttons that has been provided for the mechanical control of robotic vehicle. At the same time some keyboard icons also configured for vehicle control which are D, S, F, C, and E. By pressing Keyboard key E or command button FRONT robotic vehicle can be move forward. By pressing Keyboard key $\mathrm{C}$ or command button BACK robotic vehicle can be move backward. By pressing Keyboard key S or command button LEFT robotic vehicle can be tern left. By pressing Keyboard key F or command button RIGHT robotic vehicle can be tern right. By pressing Keyboard key D or command button STOP robotic vehicle can be move stopped. UP, DOWN, CATCH, DROP and QUIT accounts for the mechanical control of robotic arm. At the same time some keyboard icons also configured for vehicle control which are $\mathrm{J}, \mathrm{H}, \mathrm{M}$, and $\mathrm{K}, \mathrm{U}$. By using keyboard key $\mathrm{H}$ or by pressing command button $\mathrm{CATCH}$ robotic arm can move more closer, by using keyboard key $\mathrm{K}$ or by pressing command button DROP robotic arm can move more wider. By using keyboard key $\mathrm{U}$ or by pressing command button UP robotic arm can move upward. By using keyboard key $\mathrm{M}$ or by pressing command button DOWN robotic arm can move downward. By pressing Keyboard key $\mathbf{J}$ or command button QUIT robotic arm can be drop anything in their hand .All control commands sent using Bluetooth module. When the connections are to be terminated for a short while, one can use the disconnect button. On this button click, the connection between Bluetooth modules is terminated while the form will still sustain in the display. In order to re-establish a connection we can make use of connect button once more. When the application has to be terminated permanently, exit button is pressed. This will disconnect the Bluetooth module along with closing the application. 


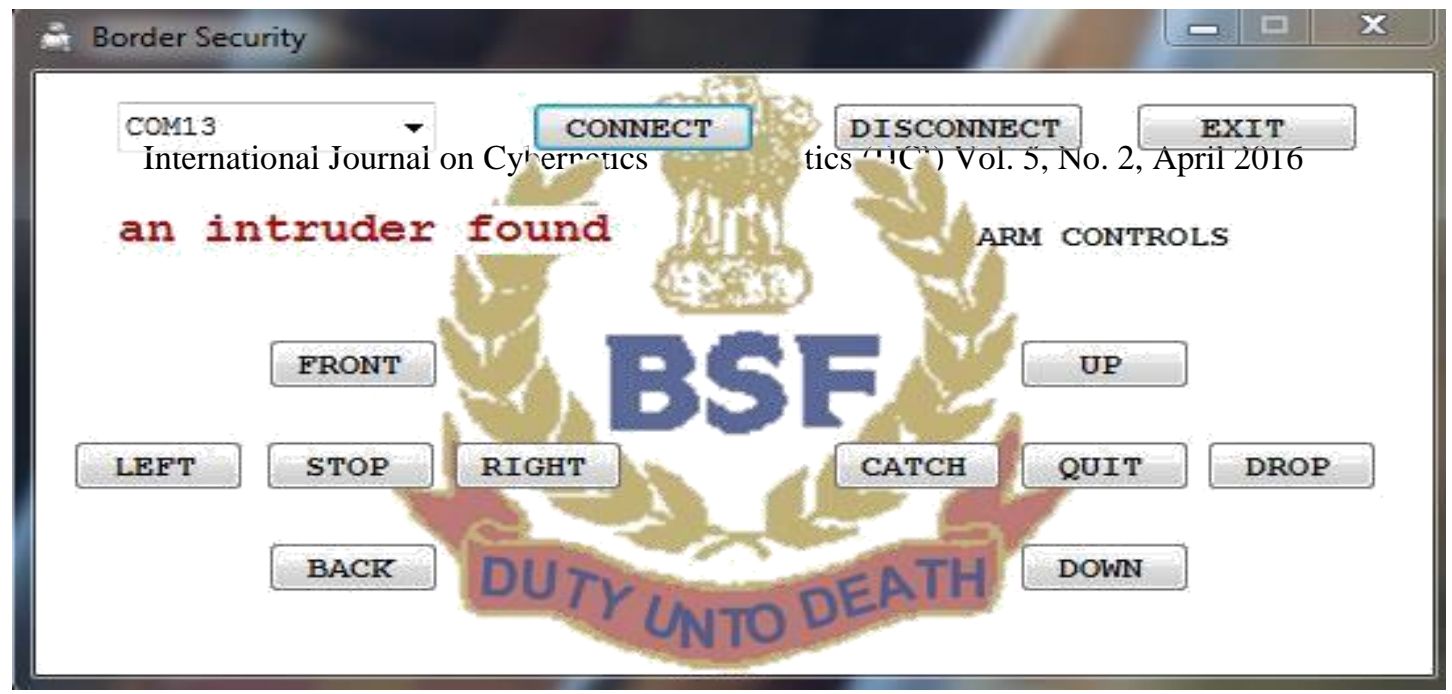

Figure 6 Notification of bomb detection

Robotic vehicle or robotic part patrolling in the borders continuously checking the presence of intruder by using PIR sensor and presence of explosive is detected by using metal detectors, which is attached in robot. Continues border scenario information is send to remote station with the help of thermal imaging camera. Infrared detector is the main part of any thermal imaging. With the help of thermal imaging camera possible to see crystal clear pictures through darkness, fog, and haze and smoke all depends on the quality of the detector. Which is a wireless camera and its receiver module is present in remote station. Receiver module receives wireless information's from thermal imaging camera and displayed in scenario monitoring PC. If any intruder is detected by robot that massage is send to remote station. "AN INTRUDER FOUND" massage is display in visual basic GUI is shown in fig.8. Similarly any explosive is detected by robot that bomb detect massage send to remote station. "BOMB DETECTED" message is display in visual basic GUI is shown in fig.7

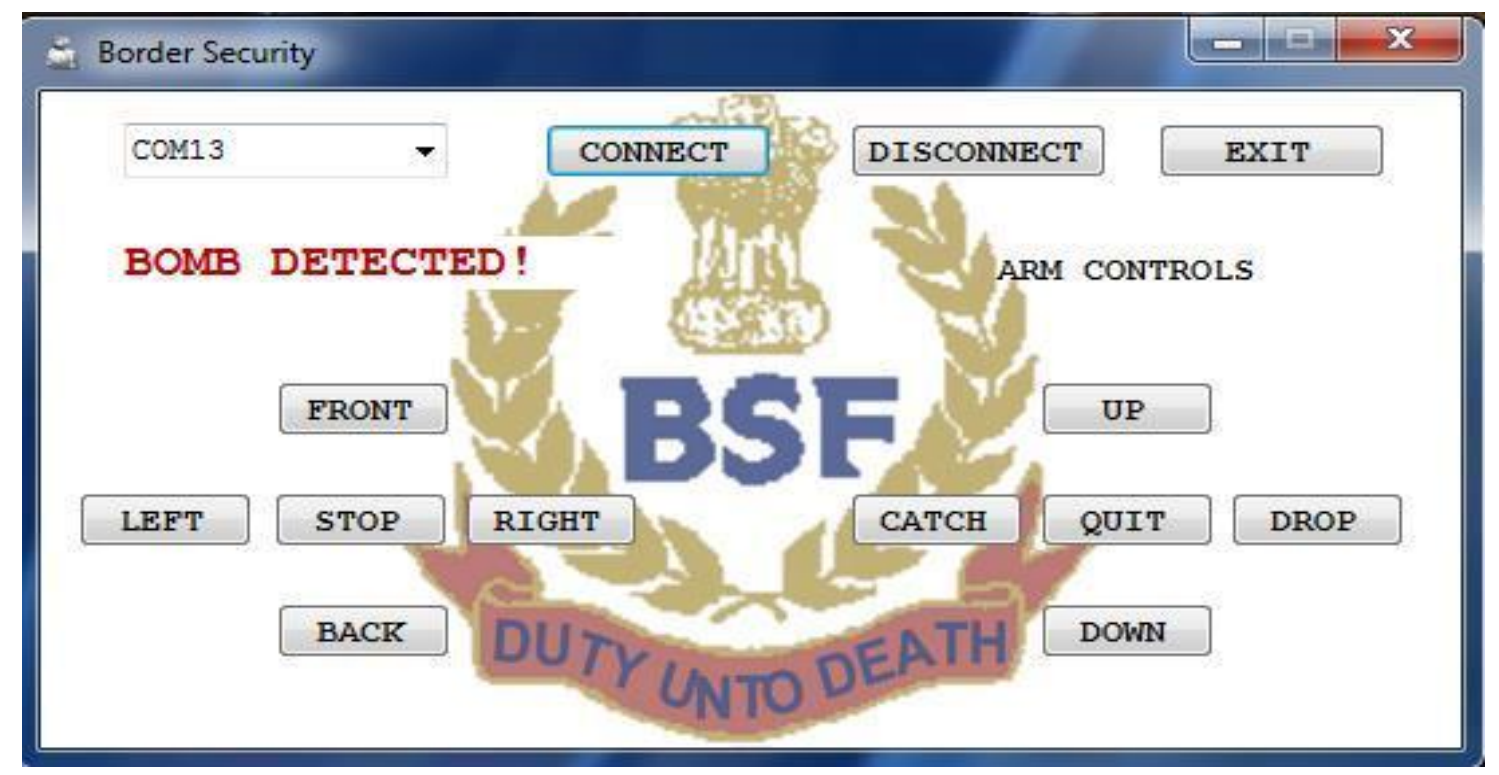

Fig.7 Notification of intruder detection 
International Journal on Cybernetics \& Informatics (IJCI) Vol. 5, No. 2, April 2016

\section{ADVANTAGES}

1. The multimedia sensors provide accurate detection as well as large detection range.

2. The thermal imaging camera provide additional information that cannot be detected by the multi-media sensors, e.g. in cases where the intruder is hidden behind an obstacle that cannot be detected

3. Mobile sensors provide intrusion tracking capability to track the intruders after they have been detected

4. By network processing, the heterogeneous sensors cooperatively detect the intrusion and report the results to a remote administrator. Accordingly, both the deployment and operational cost of the border patrol system can significantly be decreased.

\section{CONCLUSION}

At present in our country there hasn't been used a system which can automatically detect human intrusion as well as the presence of any explosive materials at the borders. Presently one or more soldiers are needed to patrol the border area in this project, we introduce Border security robot, a hybrid wireless sensor network architecture for border patrol to reduce the human involvement and used to improve the detection accuracy of current border patrol systems. The human involvement reduced with the help of PIR sensor and metal detector and thermal imaging camera. It can be concluded from the above.

\section{ACKNOWLEDGEMENT}

I take this opportunity to thank all those who have been directly or indirectly involved in making this project success. Express my honest gratitude to my project guide Prof. Aby Mathew for his constant encouragement, inspiration and guidance.

\section{REFERENCES}

[1] Zhi Sun, Pu Wang, Mehmet C,Vuran,Mznah A. Al-Rodhaan,Abdullah M.Al-Dhelaan,Ian F.Akyildiz , (2011),"BorderSense: Border patrol through advanced wireless sensor networks", Ad Hoc Networks 9 pp. $468-477$

[2] K. V. S. S. S. S. Sairam, N. Gunasekaran, S. Rama Reddy, (2002), "Bluetooth in Wireless Communication", IEEE Communications Magazine ,pp.90-96

[3] Francesco Balena ,"Programming Microsoft Visual Basic 6.0", Publisher: Microsoft Press, 1999

[4] I.F. Akyildiz, T. Melodia, K. Chowdhury,(2008) "Wireless multimedia sensor Networks : applications and testbeds", Proceedings of the IEEE 96 (10) pp. 1588-1605

[5] Ariponnammal S and Natarajan S (1994) "Control system for a mobile robot", Pramana Journal of Physics Vol.42, No:1, pp.421-425 


\section{AUTHOR}

Minni Mohan Graduated (B-tech)in Electronics and Communication Engineering from Mahathma Gandhi University and currently pursuing $M$ Tech in VLSI and Embedded System in APJ Abdul Kalam Technological University, Kerala, India.

Siddharth Shelly is a faculty member of Mar Athanasius College of Engineering, Kothamangalam, Kerala, India. He received his B.Tech from Mahatma Gandhi University, Kottayam and M.Tech degree from the Amrita School of Engineering, Coimbatore, India. His current research focus is in the area of vehicular ad hoc networks, embedded systems.

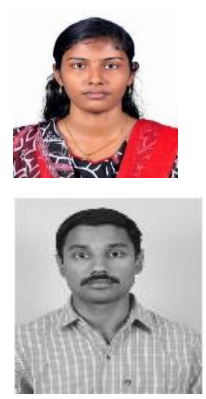

